\title{
ASYMPTOTICS FOR POLYNOMIALS SATISFYING A CERTAIN TWIN ASYMPTOTIC PERIODIC RECURRENCE RELATION: UNBOUNDED CASES*
}

\author{
E.X.L. DE ANDRADE ${ }^{\dagger}$, F.A. KUROKAWA ${ }^{\ddagger}$, AND A. SRI RANGA ${ }^{\dagger}$
}

Abstract. Polynomials satisfying a certain twin asymptotic periodic recurrence relation are considered. It is assumed that the coefficients of the recurrence formula are unbounded but vary regularly and have different behaviour for even and odd indices. The asymptotic behaviour of the ratio of contiguous polynomials is analyzed.

Key words. Orthogonal L-polynomials, Stieltjes transforms, three term recurrence relations

AMS subject classifications. Primary 42C05; Secondary 30C15, 40A30

1. Introduction. We consider the sequence of polynomials $\left\{B_{n}\right\}$ generated by the three term recurrence relation

$$
B_{n+1}(z)=\left(z-\beta_{n+1}\right) B_{n}(z)-\alpha_{n+1} z B_{n-1}(z), \quad n \geq 1,
$$

with $B_{0}(z)=1$ and $B_{1}(z)=z-\beta_{1}$, where

$$
\beta_{n}>0, \quad \alpha_{n+1}>0, \quad n \geq 1 .
$$

An important contribution on such polynomials is the paper [13] by Jones, Thron and Waadeland. They show that the zeros of $B_{n}$ are all positive, distinct and interlace with those of $B_{n-1}$. Moreover, in order to solve the strong Stieltjes moment problem, they also prove a Favard type theorem which can be stated as follows:

There exists a distribution function $\psi$ with all its points of increase within $(0, \infty)$, such that

$$
\int_{0}^{\infty} x^{-n+s} B_{n}(x) d \psi(x)=\rho_{n} \delta_{n, s}, \quad 0 \leq s \leq n, \quad n \geq 0 .
$$

Uniqueness of $\psi$ depends on the convergence of the associated T-fraction (M-fraction).

We recall that the original Favard theorem and interlacing properties of the zeros are with respect to orthogonal polynomials, in which case the three term recurrence relation is

$$
\tilde{B}_{n+1}(z)=\left(z-\tilde{\beta}_{n+1}\right) \tilde{B}_{n}(z)-\tilde{\alpha}_{n+1} \tilde{B}_{n-1}(z), \quad n \geq 1,
$$

with $\tilde{B}_{0}(z)=1$ and $\tilde{B}_{1}(z)=z-\tilde{\beta}_{1}$, where $\tilde{\beta}_{n}$ are all real and $\tilde{\alpha}_{n+1}>0$ for $n \geq 1$ (see for example [10], [16] and [22]).

A Favard type theorem for the polynomials $B_{n}$ in a somewhat more general setting than $(1.1 a)$ were also studied by Hendriksen and Van Rossum [8].

\footnotetext{
${ }^{*}$ Received October 13, 2006; accepted for publication November 9, 2007. This research was supported by grants from FAPESP, CAPES and CNPq of Brazil.

$\dagger^{\dagger}$ Departamento de Ciências de Computação e Estatística, IBILCE, Universidade Estadual Paulista, 15054-000 São José do Rio Preto, SP, Brazil (eliana@ibilce.unesp.br; ranga@ibilce.unesp. br).

¥Instituto de Ciências Matemáticas e de Computação, Universidade de São Paulo, 13560-970 São Carlos, SP, Brazil (kurokawa@icmc.usp.br).
} 
Three term recurrence relation of the type (1.1), with the restriction (1.1a) or with other restrictions, are of considerable interest in many other contexts, including two point Padé approximants, Szegő and para-orthogonal polynomials, and polynomials defined by a two dimensional difference system. See, for example, [2], [4], [12], [14], [17], [15] and [23].

In this paper we consider the asymptotic properties of the polynomials $B_{n}(z)$ assuming that recurrence coefficients $\alpha_{n}$ and $\beta_{n}$, restricted by (1.1a), are also divergent asymptotically to a regularly varying sequence as defined below.

Definition 1. A non-negative function $f: \mathbb{R}^{+} \rightarrow \mathbb{R}^{+}$is regularly varying (at infinity) if for some real $\sigma$ and all $t>0$,

$$
\lim _{x \rightarrow \infty} \frac{f(x t)}{f(x)}=t^{\sigma} .
$$

The constant $\sigma$ is called the exponent of regular variation.

If $f(x)$ is a regularly varying function with exponent $\sigma$, then $\left\{\lambda_{n}\right\}$, where $\lambda_{n}=$ $f(n)$, is called a regularly varying sequence with exponent $\sigma$.

Specifically, we give information on the limiting behaviour of the sequences of ratios $\left\{B_{n} / B_{n-2}\right\},\left\{B_{2 n-1} / B_{2 n}\right\},\left\{B_{2 n} / B_{2 n+1}\right\}$ and $\left\{B_{n}^{\prime} /\left(n B_{n}\right)\right\}$. We also apply the results to some known families of polynomials $B_{n}(z)$. A previous study of this nature, with recurrence relation (1.3), was done in [21].

Asymptotic behaviour of the ratios $\left\{B_{n} / B_{n-2}\right\},\left\{B_{2 n-1} / B_{2 n}\right\},\left\{B_{2 n} / B_{2 n+1}\right\}$ and $\left\{B_{n}^{\prime} /\left(n B_{n}\right)\right\}$, when the recurrence coefficients $\alpha_{n}$ and $\beta_{n}$ in (1.1) are bounded and converge to finite limits, is considered in [1].

2. Preliminary results. Let $K_{n}(z)=B_{n+1}^{\prime}(z) B_{n}(z)-B_{n}^{\prime}(z) B_{n+1}(z), n \geq 0$. Then from (1.1) one can establish that (see [13])

$$
K_{n}(z)=B_{n}^{2}(z)+\alpha_{n+1} \beta_{n} B_{n-1}^{2}(z)+\alpha_{n+1} \alpha_{n} z^{2} K_{n-2}(z), \quad n \geq 2,
$$

with $K_{0}(z)=B_{0}^{2}(z)=1$ and $K_{1}(z)=B_{1}^{2}(z)+\alpha_{2} \beta_{1} B_{0}^{2}(z)$. Hence from $(-1)^{n} B_{n}(0)=$ $\beta_{1} \beta_{2} \ldots \beta_{n}$ and from (1.1a) it follows that the zeros of $B_{n}$ are positive and distinct and different from those of $B_{n-1}$.

Let $z_{n, 1}<z_{n, 2}<\ldots<z_{n, n}$ be the $n$ zeros of $B_{n}$. Using the properties of chain sequences (see, for example, [3, 11]), the following result is obtained in [19]. If

$$
\hat{\beta}_{k}=\sup _{1 \leq l \leq k} \beta_{l}, \quad \check{\beta}_{k}=\inf _{1 \leq l \leq k} \beta_{l} \text { and } \quad \hat{\alpha}_{k}=\sup _{2 \leq l \leq k} \alpha_{l},
$$

for $k \geq 2$, then all the zeros of the polynomials $B_{l}, 1 \leq l \leq k$, lie inside the interval $\left[\check{d}_{k}, \hat{d}_{k}\right]$, where

$$
\hat{d}_{k}=\hat{\beta}_{k}+2 \hat{\alpha}_{k}+\sqrt{\left(\hat{\beta}_{k}+2 \hat{\alpha}_{k}\right)^{2}-\breve{\beta}_{k}^{2}}<\infty
$$

and

$$
\check{d}_{k}=\left\{\frac{1}{\check{\beta}_{k}}+\frac{2 \hat{\alpha}_{k}}{\check{\beta}_{k}^{2}}+\sqrt{\left(\frac{1}{\check{\beta}_{k}}+\frac{2 \hat{\alpha}_{k}}{\check{\beta}_{k}^{2}}\right)^{2}-\frac{1}{\hat{\beta}_{k}^{2}}}\right\}^{-1}>0 .
$$


For the partial decomposition

$$
\frac{B_{n-1}(z)}{B_{n}(z)}=\sum_{r=1}^{n} \frac{\tau_{n, r}}{z-z_{n, r}}, \quad n \geq 1
$$

we have

$$
\tau_{n, r}=\frac{B_{n-1}\left(z_{n, r}\right)}{B_{n}^{\prime}\left(z_{n, r}\right)}>0, \quad 1 \leq r \leq n, \quad \sum_{r=1}^{n} \tau_{n, r}=1 .
$$

From (1.1) we also obtain the following three term recurrences for the polynomials of even and odd indices

$$
\begin{aligned}
& B_{2 n+2}(z)=a_{2 n}(z) B_{2 n}(z)-b_{2 n}(z) B_{2 n-2}(z), \\
& B_{2 n+3}(z)=a_{2 n+1}(z) B_{2 n+1}(z)-b_{2 n+1}(z) B_{2 n-1}(z),
\end{aligned}
$$

where

$$
\begin{aligned}
a_{n}(z) & =\left(z-\beta_{n+1}\right)\left(z-\beta_{n+2}\right)-\alpha_{n+1} \frac{z-\beta_{n+2}}{z-\beta_{n}} z-\alpha_{n+2} z \\
b_{n}(z) & =\alpha_{n} \alpha_{n+1} \frac{z-\beta_{n+2}}{z-\beta_{n}} z^{2}
\end{aligned}
$$

Finally, we will be needing the following lemma, for a proof of which we refer to $[21]$.

Lemma 1. Suppose $\left\{\delta_{n, j}, j \leq n, n=1,2, \ldots\right\}$ is a bounded triangular array of complex numbers for which $\delta_{n, j} \rightarrow 0$ whenever $n \rightarrow \infty$ and $j / n \rightarrow t \in[0,1]$. Then for any $w$ with $|w|<1$,

$$
w^{k} \sum_{j=0}^{k} \delta_{n, j} w^{-j} \rightarrow 0
$$

whenever $n \rightarrow \infty$ and $j / n \rightarrow t \in[0,1]$.

3. Ratio asymptotics. From now on, we assume that there exists a regularly varying sequence $\lambda_{n}$ with exponent $\sigma>0$ such that the recurrence coefficients of (1.1), in addition to (1.1a), satisfy

$$
\begin{array}{ll}
\lim _{n \rightarrow \infty} \beta_{2 n} / \lambda_{2 n}=\beta^{(0)}, & \lim _{n \rightarrow \infty} \alpha_{2 n} / \lambda_{2 n}=\alpha^{(0)}, \\
\lim _{n \rightarrow \infty} \beta_{2 n+1} / \lambda_{2 n}=\beta^{(1)}, & \lim _{n \rightarrow \infty} \alpha_{2 n+1} / \lambda_{2 n}=\alpha^{(1)} .
\end{array}
$$

First of all, as $n \rightarrow \infty$ and $k / n \rightarrow t \in[0,1]$, we have

$$
\begin{array}{ll}
\frac{\alpha_{2 k}}{\lambda_{2 n}}=\frac{\alpha_{2 k}}{\lambda_{2 k}} \frac{\lambda_{2 k}}{\lambda_{2 n}} \rightarrow \alpha^{(0)} t^{\sigma}, & \frac{\alpha_{2 k+1}}{\lambda_{2 n}}=\frac{\alpha_{2 k+1}}{\lambda_{2 k}} \frac{\lambda_{2 k}}{\lambda_{2 n}} \rightarrow \alpha^{(1)} t^{\sigma}, \\
\frac{\beta_{2 k}}{\lambda_{2 n}}=\frac{\beta_{2 k}}{\lambda_{2 k}} \frac{\lambda_{2 k}}{\lambda_{2 n}} \rightarrow \beta^{(0)} t^{\sigma}, & \frac{\beta_{2 k+1}}{\lambda_{2 n}}=\frac{\beta_{2 k+1}}{\lambda_{2 k}} \frac{\lambda_{2 k}}{\lambda_{2 n}} \rightarrow \beta^{(1)} t^{\sigma} .
\end{array}
$$


Hence, from (2.1) and (3.2) we observe that for all $n>1$ there exists a constant $A>0$ such that

$$
0<\frac{z_{k, j}}{\lambda_{n}}<A t^{\sigma}, \quad 1 \leq j \leq k, \quad \frac{1}{n} \leq \frac{k}{n} \leq t .
$$

THEOREM 1. Let the recurrence coefficients $\alpha_{n}$ and $\beta_{n}$ in (1.1) also satisfy (3.1), where $\lambda_{n}$ is a regularly varying sequence with exponent $\sigma>0$. Let $A$ be a positive constant such that (3.3) holds. Then as $n \rightarrow \infty$ and $k / n \rightarrow t \in[0,1]$,

$$
R_{k, n}(z)=\frac{1}{\lambda_{n}^{2}} \frac{B_{k}\left(\lambda_{n} z\right)}{B_{k-2}\left(\lambda_{n} z\right)} \rightarrow R_{1}(z, t)=t^{2 \sigma} R_{1}\left(z t^{-\sigma}\right),
$$

uniformly for $z$ on compact subsets of $\mathbb{C} \backslash[0, A]$, where

$$
\begin{aligned}
R_{1}(z)=\frac{1}{2}\{( & \left.-\beta^{(0)}\right)\left(z-\beta^{(1)}\right)-\left(\alpha^{(0)}+\alpha^{(1)}\right) z \\
& \left.+\sqrt{\left.\left[\left(z-\beta^{(0)}\right)\left(z-\beta^{(1)}\right)-\left(\alpha^{(0)}+\alpha^{(1)}\right) z\right)\right]^{2}-4 \alpha^{(0)} \alpha^{(1)} z^{2}}\right\} .
\end{aligned}
$$

Proof. The theorem is clearly true if $k / n \rightarrow 0$, when we obtain $R_{1}(z, 0)=z^{2}$. Thus we assume $t>0$. Let $d>0$ and $z \in[A+d, \infty)$. Since $z-z_{k, j} / \lambda_{n}>d$ and $z_{k, j} /\left(\lambda_{n} z\right)<A /(A+d)$ for $j=1,2, \ldots k$, then for $1 \leq k \leq n$, from (2.2) and (2.3),

$$
0<\lambda_{n} \frac{B_{k-1}\left(\lambda_{n} z\right)}{B_{k}\left(\lambda_{n} z\right)}=\sum_{j=1}^{k} \frac{\tau_{k, j}}{z-z_{k, j} / \lambda_{n}}<\frac{1}{d}
$$

and

$$
0<\lambda_{n} z \frac{B_{k-1}\left(\lambda_{n} z\right)}{B_{k}\left(\lambda_{n} z\right)}=\sum_{j=1}^{k} \frac{\tau_{k, j}}{1-\frac{z_{k, j} / \lambda_{n}}{z}}<\frac{A+d}{d} .
$$

Hence,

$$
0<\left[R_{2 k, 2 n}(z)\right]^{-1}<\frac{1}{d^{2}} \quad \text { and } \quad 0<z\left[R_{2 k, 2 n}(z)\right]^{-1}<\frac{A+d}{d^{2}} .
$$

For the moment we use only the first bounding expression given in (3.6). However, different to the treatment in [21], later we will also be needing the other bounding expression.

Let $\left\{k_{n}\right\}$ be a sequence of integers such that $k_{n} / n \rightarrow t$ as $n \rightarrow \infty$. Since $\left|\left[R_{2 k_{n}, 2 n}(z)\right]^{-1}\right|$ are uniformly bounded for $z \in[A+d, \infty)$, there exists a subsequence $\left\{\left[R_{2 k_{\hat{n}}, 2 \hat{n}}(z)\right]^{-1}\right\}$ that converges to a limit function.

We now show that the subsequences $\left\{\left[R_{2 k_{\hat{n}}, 2 \hat{n}}(z)\right]^{-1}\right\}$ and $\left\{\left[R_{2 k_{\hat{n}}+2,2 \hat{n}}(z)\right]^{-1}\right\}$ have the same limit when $d$ is taken to be sufficiently large. For this, from a formula of Dombrowski [5] (see also[6] and [7]) applied to the recurrence relation (2.4), we obtain 


$$
\begin{aligned}
& \frac{B_{2 k}^{2}\left(\lambda_{2 n} z\right)-B_{2 k+2}\left(\lambda_{2 n} z\right) B_{2 k-2}\left(\lambda_{2 n} z\right)}{b_{2}\left(\lambda_{2 n} z\right) b_{4}\left(\lambda_{2 n} z\right) \cdots b_{2 k}\left(\lambda_{2 n} z\right)} \\
& =B_{0}^{2}+\sum_{j=1}^{k} \frac{a_{2 j}\left(\lambda_{2 n} z\right)-a_{2 j-2}\left(\lambda_{2 n} z\right)}{b_{2}\left(\lambda_{2 n} z\right) b_{4}\left(\lambda_{2 n} z\right) \cdots b_{2 j}\left(\lambda_{2 n} z\right)} B_{2 j}\left(\lambda_{2 n} z\right) B_{2 j-2}\left(\lambda_{2 n} z\right) \\
& \quad-\sum_{j=1}^{k} \frac{b_{2 j}\left(\lambda_{2 n} z\right)-b_{2 j-2}\left(\lambda_{2 n} z\right)}{b_{2}\left(\lambda_{2 n} z\right) b_{4}\left(\lambda_{2 n} z\right) \cdots b_{2 j}\left(\lambda_{2 n} z\right)} B_{2 j}\left(\lambda_{2 n} z\right) B_{2 j-4}\left(\lambda_{2 n} z\right) .
\end{aligned}
$$

From this,

$$
\begin{aligned}
& \left|\left[R_{2 k+2,2 n}(z)\right]^{-1}-\left[R_{2 k, 2 n}(z)\right]^{-1}\right| \\
& \leq \lambda_{2 n}^{2}\left|\frac{\tilde{b}_{2}\left(\lambda_{2 n} z\right) \tilde{b}_{4}\left(\lambda_{2 n} z\right) \cdots \tilde{b}_{2 k}\left(\lambda_{2 n} z\right)}{\lambda_{2 n}^{2 k}}\right| \frac{\left|\lambda_{2 n}^{2} z\right|^{2 k}}{\left|B_{2 k+2}\left(\lambda_{2 n} z\right) B_{2 k}\left(\lambda_{2 n} z\right)\right|} \\
& \quad+\lambda_{2 n}^{2} \sum_{j=0}^{k}\left|\frac{\tilde{b}_{2 j+2}\left(\lambda_{2 n} z\right) \cdots \tilde{b}_{2 k}\left(\lambda_{2 n} z\right)}{\lambda_{2 n}^{2(k-j)}}\right|\left|\lambda_{2 n}^{2} z\right|^{2(n-j)}\left\{U_{n, j}(z)+V_{n, j}(z)\right\},
\end{aligned}
$$

where $\tilde{b}_{2 j}\left(\lambda_{2 n} z\right)=b_{2 j}\left(\lambda_{2 n} z\right) /\left(\lambda_{2 n}^{2} z^{2}\right), U_{n, j}(z)=u_{n, j}(z)\left|\frac{B_{2 j}\left(\lambda_{2 n} z\right) B_{2 j-2}\left(\lambda_{2 n} z\right)}{B_{2 k+2}\left(\lambda_{2 n} z\right) B_{2 k}\left(\lambda_{2 n} z\right)}\right|$ and $V_{n, j}(z)=v_{n, j}(z)\left|\frac{B_{2 j}\left(\lambda_{2 n} z\right) B_{2 j-4}\left(\lambda_{2 n} z\right)}{B_{2 k+2}\left(\lambda_{2 n} z\right) B_{2 k}\left(\lambda_{2 n} z\right)}\right|$, with $u_{n, j}(z)=\left|a_{2 j}\left(\lambda_{2 n} z\right)-a_{2 j-2}\left(\lambda_{2 n} z\right)\right|$ and $v_{n, j}(z)=\left|b_{2 j}\left(\lambda_{2 n} z\right)-b_{2 j-2}\left(\lambda_{2 n} z\right)\right|$.

From (3.2), for our sequence $\left\{k_{n}\right\}$ the array $\left\{\tilde{b}_{2 j}\left(\lambda_{2 n} z\right) / \lambda_{2 n}^{2} ; j \leq k_{n}, n=\right.$ $1,2, \ldots\}$ is bounded by a constant $C$. Also, for $j \leq k$, by means of the two bounding expressions in (3.6),

$$
\left|\frac{B_{2 j}\left(\lambda_{2 n} z\right)}{B_{2 k}\left(\lambda_{2 n} z\right)}\right|=\prod_{i=j+1}^{k}\left|\frac{B_{2 i-2}\left(\lambda_{2 n} z\right)}{B_{2 i}\left(\lambda_{2 n} z\right)}\right|<\left(\frac{1}{\lambda_{2 n} d}\right)^{2 k-2 j}
$$

and

$$
\left|z^{k-j} \frac{B_{2 j}\left(\lambda_{2 n} z\right)}{B_{2 k}\left(\lambda_{2 n} z\right)}\right|=\prod_{i=j+1}^{k}\left|z \frac{B_{2 i-2}\left(\lambda_{2 n} z\right)}{B_{2 i}\left(\lambda_{2 n} z\right)}\right|<\left(\frac{A+d}{\lambda_{2 n}^{2} d^{2}}\right)^{k-j} .
$$

Hence, (3.7) leads to

$$
\left|\left[R_{2 k+2,2 n}(z)\right]^{-1}-\left[R_{2 k, 2 n}(z)\right]^{-1}\right| \leq \frac{1}{d^{2}} w^{k}+w^{k} \sum_{j=1}^{k} \delta_{n, j}(z) w^{-j} .
$$

where $w=\frac{C(A+d)^{2}}{d^{4}}$ and $\delta_{n, j}(z)=\frac{\left|u_{n, j}(z)\right|}{\lambda_{2 n}^{2} d^{4}}+\frac{\left|v_{n, j}(z)\right|}{\lambda_{2 n}^{4} d^{6}}$.

Now we choose $d=D$ large enough so that $w<1$. Thus, by Lemma 1 it follows that for $n \rightarrow \infty$ and $k / n \rightarrow t \in[0,1]$, if $z \in[A+D, \infty)$, then

$$
\left|\left[R_{2 k+2,2 n}(z)\right]^{-1}-\left[R_{2 k, 2 n}(z)\right]^{-1}\right| \rightarrow 0 .
$$


Hence for $z \in[A+D, \infty)$, if $\left\{\left[R_{2 k_{\hat{n}}, 2 \hat{n}}(z)\right]^{-1}\right\}$ is a subsequence that converges, then also $\left\{\left[R_{2 k_{\hat{n}}+2,2 \hat{n}}(z)\right]^{-1}\right\}$ and they both have the same limit. Let this limit be $1 / r(z, t)$.

From the recurrence formula in (2.4) we have

$$
R_{2 k_{\hat{n}}+2,2 \hat{n}}(z)=\frac{a_{2 k_{\hat{n}}}\left(\lambda_{2 \hat{n}} z\right)}{\lambda_{2 \hat{n}}^{2}}-\frac{b_{2 k_{\hat{n}}}\left(\lambda_{2 \hat{n}} z\right)}{\lambda_{2 \hat{n}}^{4}}\left[R_{2 k_{\hat{n}}, 2 \hat{n}}(z)\right]^{-1} .
$$

Hence, in the limit,

$$
r(z, t)=a(z, t)-\frac{b(z, t)}{r(z, t)}
$$

where $a(z, t)=\left(z-\beta^{(0)} t^{\sigma}\right)\left(z-\beta^{(1)} t^{\sigma}\right)-\left(\alpha^{(0)}+\alpha^{(1)}\right) t^{\sigma} z$ and $b(z, t)=\alpha^{(0)} \alpha^{(1)} t^{2 \sigma} z^{2}$.

From this, also with the observation $\lim _{z \rightarrow \infty} R_{2 k_{\hat{n}}, 2 \hat{n}}(z)=\infty$, it follows that

$$
r(z, t)=R_{1}(z, t)=\frac{1}{2}\left\{a(z, t)+\sqrt{a^{2}(z, t)-4 b(z, t)}\right\} .
$$

Note that, from (3.8) and (3.9), every convergent subsequence $\left\{\left[R_{2 k_{\hat{n}}, 2 \hat{n}}(z)\right]^{-1}\right\}$ has the same limit $R_{1}(z, t)$. Thus we conclude that, for any $z \in[A+D, \infty)$,

$$
R_{2 k_{n}, 2 n}(z) \rightarrow R_{1}(z, t),
$$

as $n \rightarrow \infty$ and $k_{n} / n \rightarrow t \in[0,1]$.

However, $\left\{\left[R_{2 k_{n}, 2 n}(z)\right]^{-1}\right\}$ is a sequence of analytic functions on $\mathbb{C} \backslash[0, A]$. Let $K$ be a compact subset in $\mathbb{C} \backslash[0, A]$. Let $\delta$ be the distance from $K$ to the interval $[0, A]$. Since $\delta$ is strictly positive, we also have from (3.5) that

$$
\left|\left[R_{2 k_{n}, 2 n}(z)\right]^{-1}\right| \leq \frac{1}{\delta^{2}} \quad \text { for } \quad z \in K
$$

Hence, by the Stieltjes-Vitali theorem (see $[9]$ ), $\left\{\left[R_{2 k_{n}, 2 n}(z)\right]^{-1}\right\}$ converges to $1 / R_{1}(z, t)$ uniformly on compact subsets of $\mathbb{C} \backslash[0, A]$.

Now if we repeat the same reasoning with the sequence $\left\{\left[R_{2 k_{n}+1,2 n}(z)\right]^{-1}\right\}$, we find that this sequence also converges to $1 / R_{1}(z, t)$ and this completes the proof.

From the recurrence relation (1.1) we obtain

$$
\frac{1}{\lambda_{2 n}^{2}} \frac{B_{2 k+1}\left(\lambda_{2 n} z\right)}{B_{2 k-1}\left(\lambda_{2 n} z\right)}=\left(z-\frac{\beta_{2 k+1}}{\lambda_{2 n}}\right) \frac{1}{\lambda_{2 n}} \frac{B_{2 k}\left(\lambda_{2 n} z\right)}{B_{2 k-1}\left(\lambda_{2 n} z\right)}-\frac{\alpha_{2 k+1}}{\lambda_{2 n}} z
$$

and

$$
\frac{1}{\lambda_{2 n}^{2}} \frac{B_{2 k+2}\left(\lambda_{2 n} z\right)}{B_{2 k}\left(\lambda_{2 n} z\right)}=\left(z-\frac{\beta_{2 k+2}}{\lambda_{2 n}}\right) \frac{1}{\lambda_{2 n}} \frac{B_{2 k+1}\left(\lambda_{2 n} z\right)}{B_{2 k}\left(\lambda_{2 n} z\right)}-\frac{\alpha_{2 k+2}}{\lambda_{2 n}} z .
$$

Letting $n \rightarrow \infty$ and $k / n \rightarrow t$, then from Theorem 1 we have the following result.

TheOrem 2. With the conditions of Theorem 1 , as $n \rightarrow \infty$ and $k / n \rightarrow t \in[0,1]$,

$$
\begin{aligned}
& \lambda_{2 n} \frac{B_{2 k}\left(\lambda_{2 n} z\right)}{B_{2 k+1}\left(\lambda_{2 n} z\right)} \rightarrow R_{2}^{(0)}(z, t)=\frac{z-\beta^{(0)} t^{\sigma}}{R_{1}(z, t)+\alpha^{(0)} t^{\sigma} z}, \\
& \lambda_{2 n} \frac{B_{2 k-1}\left(\lambda_{2 n} z\right)}{B_{2 k}\left(\lambda_{2 n} z\right)} \rightarrow R_{2}^{(1)}(z, t)=\frac{z-\beta^{(1)} t^{\sigma}}{R_{1}(z, t)+\alpha^{(1)} t^{\sigma} z},
\end{aligned}
$$


uniformly on compact sets of $\mathbb{C} \backslash[0, A]$.

Now the initial part of the proof of the next theorem is exactly as in Theorem 3 of [21]. To complete the proof, from

$$
2 R_{1}(z, t)=z^{2}-u_{1} t^{\sigma} z+u_{2} t^{2 \sigma}+\sqrt{\left[z^{2}-u_{1} t^{\sigma} z+u_{2} t^{2 \sigma}\right]^{2}-4 u_{3} t^{2 \sigma} z^{2}},
$$

where $u_{1}=\beta^{(0)}+\beta^{(1)}+\alpha^{(0)}+\alpha^{(1)}, \quad u_{2}=\beta^{(0)} \beta^{(1)}$ and $u_{3}=\alpha^{(0)} \alpha^{(1)}$, we observe that

$$
\frac{\partial}{\partial z} R_{1}(z, t)=\frac{R_{1}(z, t)\left\{2 z-u_{1} t^{\sigma}-\frac{2 u_{3} t^{2 \sigma} z}{R_{1}(z, t)}\right\}}{\sqrt{\left[z^{2}-u_{1} t^{\sigma} z+u_{2} t^{2 \sigma}\right]^{2}-4 u_{3} t^{2 \sigma} z^{2}}} .
$$

With the use of (3.10), this leads to

$$
\frac{(\partial / \partial z) R_{1}(z, t)}{2 R_{1}(z, t)}=R_{3}(z, t)=\frac{1}{2 z}+\frac{1}{2 z} \frac{z^{2}-u_{2} t^{2 \sigma}}{\sqrt{\left[z^{2}-u_{1} t^{\sigma} z+u_{2} t^{2 \sigma}\right]^{2}-4 u_{3} t^{2 \sigma} z^{2}}} .
$$

Theorem 3. Suppose that the conditions of Theorem 1 hold. Then, as $n \rightarrow \infty$, we have

$$
\begin{aligned}
\frac{d B_{n}\left(\lambda_{n} z\right) / d z}{n B_{n}\left(\lambda_{n} z\right)} & \rightarrow R_{4}(z)=\int_{0}^{1} R_{3}(z, t) d t \\
& =\int_{0}^{1}\left[\frac{1}{2 z}+\frac{1}{2 z} \frac{z^{2}-\beta^{(0)} \beta^{(1)} t^{2 \sigma}}{\sqrt{\left(z-a t^{\sigma}\right)\left(z-\tilde{a} t^{\sigma}\right)\left(z-\tilde{b} t^{\sigma}\right)\left(z-b t^{\sigma}\right)}}\right] d t
\end{aligned}
$$

uniformly on compact subsets of $\mathbb{C} \backslash[0, A]$, where $0 \leq a \leq \tilde{a} \leq \tilde{b} \leq b$ are such that

$$
\begin{aligned}
& a+b=\beta^{(0)}+\beta^{(1)}+\alpha^{(0)}+\alpha^{(1)}+2 \sqrt{\alpha^{(0)} \alpha^{(1)}}, \\
& \tilde{a}+\tilde{b}=\beta^{(0)}+\beta^{(1)}+\alpha^{(0)}+\alpha^{(1)}-2 \sqrt{\alpha^{(0)} \alpha^{(1)}}
\end{aligned}
$$

and $a b=\tilde{a} \tilde{b}=\beta^{(0)} \beta^{(1)}$.

4. Integral representations and Quadrature rules. First of all, to be able to obtain the results given in Theorems 4 and 5 of this section and the results associated with case 2 of section 5 , we require the two lemmas given below.

Lemma 2. Let $\delta, d_{1}$ and $d_{2}$ be such that $0 \leq \delta \leq d_{1}<d_{2}$. Let $g$ be the probability distribution function defined on $\left[d_{1}^{2}, d_{2}^{2}\right]$ by

$$
g\left(x ; d_{1}, d_{2}, \delta\right)=\chi \int_{d_{1}^{2}}^{x} \frac{\sqrt{d_{2}^{2}-u} \sqrt{u-d_{1}^{2}}}{u-\delta^{2}} d u,
$$

where $\chi=\frac{2}{\pi} \frac{\left[\left(d_{2}^{2}-\delta^{2}\right)^{1 / 2}+\left(d_{1}^{2}-\delta^{2}\right)^{1 / 2}\right]^{2}}{\left(d_{2}^{2}-d_{1}^{2}\right)^{2}}$. Then

$$
\begin{aligned}
\int_{d_{1}^{2}}^{d_{2}^{2}} \frac{d g\left(x ; d_{1}, d_{2}, \delta\right)}{z-x} & =\frac{2}{z-\delta^{2}-\left[\left(d_{2}^{2}-\delta^{2}\right)\left(d_{1}^{2}-\delta^{2}\right)\right]^{1 / 2}+\sqrt{z-d_{1}^{2}} \sqrt{z-d_{2}^{2}}} \\
& =\frac{2\left[z-\delta^{2}-\left[\left(d_{2}^{2}-\delta^{2}\right)\left(d_{1}^{2}-\delta^{2}\right)\right]^{1 / 2}-\sqrt{z-d_{1}^{2}} \sqrt{z-d_{2}^{2}}\right]}{\left[\left(d_{2}^{2}-\delta^{2}\right)^{1 / 2}-\left(d_{1}^{2}-\delta^{2}\right)^{1 / 2}\right]^{2}\left(z-\delta^{2}\right)} .
\end{aligned}
$$


A proof of this easily follows from a lemma given in [20].

From Lemma 2, one also obtains

$$
\begin{aligned}
\int_{d_{1}^{2}}^{d_{2}^{2}} \frac{d g\left(x ; d_{1}, d_{2}, 0\right)}{z-x}= & \frac{2\left[-d_{1} d_{2}+\delta^{2}+\left[\left(d_{2}^{2}-\delta^{2}\right)\left(d_{1}^{2}-\delta^{2}\right)\right]^{1 / 2}\right]}{\left[d_{2}-d_{1}\right]^{2}} \frac{1}{z} \\
& +\frac{\left[\left(d_{2}^{2}-\delta^{2}\right)^{1 / 2}-\left(d_{1}^{2}-\delta^{2}\right)^{1 / 2}\right]^{2}}{\left[d_{2}-d_{1}\right]^{2}} \frac{z-\delta^{2}}{z} \int_{d_{1}^{2}}^{d_{2}^{2}} \frac{d g\left(x ; d_{1}, d_{2}, \delta\right)}{z-x} .
\end{aligned}
$$

Now let $\beta, b_{1}$ and $b_{2}$ be such that $0<\beta \leq b_{1}<b_{2}$. Then there exist unique real numbers $d_{1}$ and $d_{2}$ such that

$$
0 \leq d_{1}<d_{2} \quad \text { and } \quad d_{i}=\sqrt{b_{i}}-\beta / \sqrt{b_{i}}, i=1,2 .
$$

Here the (one to one) relation between $b_{i}$ and $d_{i}$ is such that $\left(z-b_{i}\right)\left(z-\beta^{2} / b_{i}\right)=$ $(z-\beta)^{2}-d_{i}^{2} z$.

Let $I_{E\left(\beta, b_{1}, b_{2}\right)}$ be the indicator function of the set $E\left(\beta, b_{1}, b_{2}\right)=\left[\beta^{2} / b_{2}, \beta^{2} / b_{1}\right] \cup$ $\left[b_{1}, b_{2}\right]$. That is, $I_{E\left(\beta, b_{1}, b_{2}\right)}(x)=U\left(x-\beta^{2} / b_{2}\right)-U\left(x-\beta^{2} / b_{1}\right)+U\left(x-b_{1}\right)-U\left(x-b_{2}\right)$, where $U(x)$ is the Heaviside function

$$
U(x)= \begin{cases}1, & x \geq 0 \\ 0, & x<0\end{cases}
$$

Then we can state the following lemma regarding the Stieltjes transforms of the distribution functions $F$ and $G$ supported on $E\left(\beta, b_{1}, b_{2}\right)$.

Lemma 3. For the probability distribution function $F$ defined by

$$
F\left(x ; \beta, d_{1}, d_{2}\right)=\frac{1}{2 \pi} \int_{0}^{x} \frac{|u-\beta|(u+\beta) / u}{\sqrt{d_{2}^{2} u-(u-\beta)^{2}} \sqrt{(u-\beta)^{2}-d_{1}^{2} u}} I_{E}(u) d u
$$

holds

$$
S(F ; z)=\int_{E\left(\beta, b_{1}, b_{2}\right)} \frac{d F\left(x ; \beta, d_{1}, d_{2}\right)}{z-x}=\frac{1}{2 z}+\frac{1}{2 z} \frac{z^{2}-\beta^{2}}{\sqrt{(z-\beta)^{2}-d_{1}^{2} z} \sqrt{(z-\beta)^{2}-d_{2}^{2} z}} .
$$

Moreover, if $\chi=\frac{2}{\pi} \frac{\left[\left(d_{2}^{2}-\delta^{2}\right)^{1 / 2}+\left(d_{1}^{2}-\delta^{2}\right)^{1 / 2}\right]^{2}}{\left(d_{2}^{2}-d_{1}^{2}\right)^{2}}$ and if $\delta$ and $\tau$ are such that $0 \leq \delta \leq d_{1}$ and $\beta^{2} / b_{1} \leq \tau \leq b_{1}$, then for the probability distribution function $G$ defined by

$$
\begin{aligned}
& G\left(x ; \beta, d_{1}, d_{2} ; \delta, \tau\right) \\
& \quad=\chi \int_{0}^{x} \frac{\sqrt{d_{2}^{2} u-(u-\beta)^{2}} \sqrt{(u-\beta)^{2}-d_{1}^{2} u}}{\left[(u-\beta)^{2}-\delta^{2} u\right]} \frac{(u-\beta)(u-\tau)}{|u-\beta| u} I_{E}(u) d u,
\end{aligned}
$$

holds

$$
\begin{aligned}
& S(G ; z)=\int_{E\left(\beta, b_{1}, b_{2}\right)} \frac{d G\left(x ; \beta, d_{1}, d_{2} ; \delta, \tau\right)}{z-x}=L\left(z ; \beta, d_{1}, d_{2} ; \delta, \tau\right) \\
& =\frac{2(z-\tau)}{\left[(z-\beta)^{2}-\delta^{2} z\right]-\left[\left(d_{2}^{2}-\delta^{2}\right)\left(d_{1}^{2}-\delta^{2}\right)\right]^{1 / 2} z+\sqrt{(z-\beta)^{2}-d_{1}^{2} z} \sqrt{(z-\beta)^{2}-d_{2}^{2} z}} .
\end{aligned}
$$

The proof of this lemma follows from Lemma 2 as shown in [1]. 
TheOrem 4. Suppose that (3.1) holds with $\beta^{(0)} \beta^{(1)}>0$ and $\alpha^{(0)} \alpha^{(1)}>0$. Then for the functions $R_{2}^{(0)}(z, t), R_{2}^{(1)}(z, t)$ and $R_{3}(z, t)$ obtained in Theorem 2 and Theorem 3,

$R_{2}^{(0)}(z, t)=t^{-\sigma} R_{2}^{(0)}\left(z t^{-\sigma}\right), R_{2}^{(1)}(z, t)=t^{-\sigma} R_{2}^{(1)}\left(z t^{-\sigma}\right)$ and $R_{3}(z, t)=t^{-\sigma} R_{3}\left(z t^{-\sigma}\right)$, where, for $i=0,1$,

$$
R_{2}^{(i)}(z)=\frac{1-\alpha_{\min } / \alpha^{(i)}}{z-\beta^{(1-i)}}+\frac{\alpha_{\min }}{\alpha^{(i)}} \int_{E\left(\beta, b_{1}, b_{2}\right)} \frac{d G\left(x ; \beta, d_{1}, d_{2} ; \delta, \beta^{(i)}\right)}{z-x},
$$

and

$$
R_{3}(z)=\int_{E\left(\beta, b_{1}, b_{2}\right)} \frac{d F\left(x ; \beta, d_{1}, d_{2}\right)}{z-x},
$$

with $\alpha_{\text {min }}=\min \left\{\alpha^{(0)}, \alpha^{(1)}\right\}, \quad \beta^{2}=\beta^{(0)} \beta^{(1)}, \quad \delta^{2}=\left(\sqrt{\beta^{(0)}}-\sqrt{\beta^{(1)}}\right)^{2}$,

$$
\left(\sqrt{b_{1}}-\beta / \sqrt{b_{1}}\right)^{2}=d_{1}^{2}=\delta^{2}+\left(\sqrt{\alpha^{(0)}}-\sqrt{\alpha^{(1)}}\right)^{2}
$$

and

$$
\left(\sqrt{b_{2}}-\beta / \sqrt{b_{2}}\right)^{2}=d_{2}^{2}=\delta^{2}+\left(\sqrt{\alpha^{(0)}}+\sqrt{\alpha^{(1)}}\right)^{2} .
$$

Proof. From (3.11) and Theorem 1 we obtain that $R_{2}^{(i)}(z, t)=t^{-\sigma} R_{2}^{(i)}\left(z t^{-\sigma}\right)$, where

$$
\begin{aligned}
R_{2}^{(i)}(z) & =\frac{z-\beta^{(i)}}{R_{1}(z)+\alpha^{(i)} z} \\
& =\frac{2\left(z-\beta^{(i)}\right)}{\left[(z-\beta)^{2}-\delta^{2} z\right]+\left[\alpha^{(i)}-\alpha^{(1-i)}\right] z+\sqrt{(z-\beta)^{2}-d_{1}^{2} z} \sqrt{(z-\beta)^{2}-d_{2}^{2} z}} .
\end{aligned}
$$

From this we find that

$$
\begin{aligned}
R_{2}^{(i)}(z)=\frac{\left|\alpha^{(1-i)}-\alpha^{(i)}\right|-\left(\alpha^{(1-i)}-\alpha^{(i)}\right)}{2 \alpha^{(i)}\left(z-\beta^{(1-i)}\right)} \\
\quad+\frac{\left(\alpha^{(1-i)}+\alpha^{(i)}\right)-\left|\alpha^{(1-i)}-\alpha^{(i)}\right|}{2 \alpha^{(i)}} L\left(z ; \beta, d_{1}, d_{2} ; \delta, \beta^{(i)}\right) .
\end{aligned}
$$

Hence the first result of the theorem follows from Equation (4.4) in Lemma 3.

Finally, since $R_{3}(z)$ can be written as

$$
R_{3}(z)=\frac{1}{2 z}+\frac{1}{2 z} \frac{z^{2}-\beta^{2}}{\sqrt{(z-\beta)^{2}-d_{1}^{2} z} \sqrt{(z-\beta)^{2}-d_{2}^{2} z}} .
$$

the last result of the theorem is obtained from (4.3) in Lemma 3 .

Note that one can also write

$$
\begin{aligned}
R_{2}^{(i)}(z, t)= & \frac{1-\alpha_{\min } / \alpha^{(i)}}{z-\beta^{(1-i)} t^{\sigma}}+\frac{\alpha_{\min }}{\alpha^{(i)}} \int_{E\left(\beta, b_{1}, b_{2}\right)} \frac{d G\left(x ; \beta, d_{1}, d_{2} ; \delta, \beta^{(i)}\right)}{z-x t^{\sigma}} \\
= & \frac{1-\alpha_{\min } / \alpha^{(i)}}{z-\beta^{(1-i)} t^{\sigma}} \\
& \quad+\frac{\alpha_{\min }}{\alpha^{(i)}} \int_{E\left(\beta t^{\sigma}, b_{1} t^{\sigma}, b_{2} t^{\sigma}\right)} \frac{d G\left(x ; \beta t^{\sigma}, d_{1} t^{\sigma / 2}, d_{2} t^{\sigma / 2} ; \delta t^{\sigma / 2}, \beta^{(i)} t^{\sigma}\right)}{z-x},
\end{aligned}
$$


for $i=0,1$, and

$$
R_{3}(z, t)=\int_{E\left(\beta, b_{1}, b_{2}\right)} \frac{d F\left(x ; \beta, d_{1}, d_{2}\right)}{z-x t^{\sigma}}=\int_{E\left(\beta t^{\sigma}, b_{1} t^{\sigma}, b_{2} t^{\sigma}\right)} \frac{d F\left(x ; \beta t^{\sigma}, d_{1} t^{\sigma / 2}, d_{2} t^{\sigma / 2}\right)}{z-x} .
$$

Theorem 5. Suppose that (3.1) holds with $\beta^{(0)} \beta^{(1)}>0$ and $\alpha^{(0)} \alpha^{(1)}>0$. As $n \rightarrow \infty$ and $k / n \rightarrow t \in[0,1]$, for every continuous function $f$ on $(0, \infty)$, we have

$$
\begin{aligned}
\sum_{j=1}^{2 k+i} \tau_{2 k+i, j} f\left(\frac{z_{2 k+i, j}}{\lambda_{2 n}}\right) \rightarrow & \frac{\alpha^{(1-i)}-\alpha_{\min }}{\alpha^{(1-i)}} f\left(\beta^{(i)} t^{\sigma}\right) \\
& \quad+\frac{\alpha_{\min }}{\alpha^{(1-i)}} \int_{E\left(\beta, b_{1}, b_{2}\right)} f\left(x t^{\sigma}\right) d G\left(x ; \beta, d_{1}, d_{2} ; \delta, \beta^{(1-i)}\right),
\end{aligned}
$$

for $i=0,1$, and

$$
\frac{1}{n} \sum_{j=1}^{n} f\left(\frac{z_{n, j}}{\lambda_{n}}\right) \rightarrow \int_{0}^{1} \int_{E\left(\beta, b_{1}, b_{2}\right)} f\left(x t^{\sigma}\right) d F\left(x ; \beta, d_{1}, d_{2}\right) d t,
$$

where $\beta, b_{1}, b_{2}, d_{1}, d_{2}$ and $\delta$ are as in Theorem 4.

Proof. Consider the discrete distribution functions

$$
G_{n, k}(x)=\sum_{j=1}^{k} \tau_{k, j} U\left(x-z_{k, j} / \lambda_{n}\right)
$$

and

$$
F_{n}(x)=\frac{1}{n} \sum_{j=1}^{n} U\left(x-z_{n, j} / \lambda_{n}\right)
$$

where $U(x)$ is the Heaviside function (4.2). Since

$$
\lambda_{n} \frac{B_{k-1}\left(\lambda_{n} z\right)}{B_{k}\left(\lambda_{n} z\right)}=\sum_{j=1}^{k} \frac{\tau_{k, j}}{z-z_{k, j} / \lambda_{n}} \quad \text { and } \quad \frac{1}{n} \frac{(d / d z) B_{n}\left(\lambda_{n} z\right)}{B_{n}\left(\lambda_{n} z\right)}=\frac{1}{n} \sum_{j=1}^{n} \frac{1}{z-z_{n, j} / \lambda_{n}},
$$

the Stieltjes transforms of these distribution functions are

$$
S\left(G_{n, k} ; z\right)=\int_{-\infty}^{\infty} \frac{d G_{n, k}(x)}{z-x}=\lambda_{n} \frac{B_{k-1}\left(\lambda_{n} z\right)}{B_{k}\left(\lambda_{n} z\right)}
$$

and

$$
S\left(F_{n} ; z\right)=\int_{-\infty}^{\infty} \frac{d F_{n}(x)}{z-x}=\frac{1}{n} \frac{(d / d z) B_{n}\left(\lambda_{n} z\right)}{B_{n}\left(\lambda_{n} z\right)}
$$

The asymptotic behaviour of these Stieltjes transforms is given by Theorems 2 and 3. Now, by Grommer-Hamburger theorem, the limits have to be the Stieltjes transforms of the weak limits of the distribution functions $G_{n, k}(x)$ and $F_{n}(x)$. The identification of the limits of (4.9) and (4.10) can be done by using (4.5) and (4.6). $\mathrm{\square}$ 
5. Special cases. We now consider some especial cases. Note that, even though the coefficients $\beta_{n}, \alpha_{n+1}$ are positive, any of $\beta^{(0)}, \alpha^{(0)}, \beta^{(1)}$ and $\alpha^{(1)}$ can assume the value zero.

Case 1. First we consider the case $\alpha^{(0)}=\alpha^{(1)}=\alpha>0$ and $\beta^{(0)}=\beta^{(1)}=\beta>0$.

Then in Theorem $4, \delta=d_{1}=0, d_{2}=2 \sqrt{\alpha}, \beta=b_{1}$ and $b_{2}=\beta+2 \alpha+$ $\sqrt{(\beta+2 \alpha)^{2}-\beta^{2}}$. Thus, for example, the results of Theorem 5 can be stated as

$$
\begin{aligned}
\sum_{j=1}^{k} \tau_{k, j} f\left(\frac{z_{k, j}}{\lambda_{n}}\right) \rightarrow & \int_{E\left(\beta, \beta, b_{2}\right)} f\left(x t^{\sigma}\right) d G(x ; \beta, 0,2 \sqrt{\alpha} ; 0, \beta) \\
& =\frac{1}{2 \pi \alpha} \int_{\beta_{2} / b_{2}}^{b_{2}} f\left(x t^{\sigma}\right) \frac{\sqrt{4 \alpha x-(x-\beta)^{2}}}{x} d x \\
\frac{1}{n} \sum_{j=1}^{n} f\left(\frac{z_{n, j}}{\lambda_{n}}\right) \rightarrow & \int_{0}^{1} \int_{E\left(\beta, \beta, b_{2}\right)} f\left(x t^{\sigma}\right) d F(x ; \beta, 0,2 \sqrt{\alpha}) \\
& =\frac{1}{2 \pi} \int_{0}^{1} \int_{\beta^{2} / b_{2}}^{b_{2}} f\left(x t^{\sigma}\right) \frac{1+\beta / x}{\sqrt{4 \alpha x-(x-\beta)^{2}}} d x d t
\end{aligned}
$$

Case 2. Another important case, not covered by the previous theorems, is when one of $\beta^{(0)}$ or $\beta^{(1)}$ is equal to zero. That is, for $r$ equal to 0 or 1 ,

$$
\alpha^{(r)}>0, \alpha^{(1-r)}>0, \beta^{(r)}=0 \text { and } \beta^{(1-r)}=\tilde{\beta} \geq 0 .
$$

All the explicitly given examples which are known to us so far belong to this case.

It follows that $\beta=0, \delta^{2}=\tilde{\beta}, b_{1}=d_{1}^{2}=\tilde{\beta}+\left(\sqrt{\alpha^{(0)}}-\sqrt{\alpha^{(1)}}\right)^{2}$ and $b_{2}=d_{2}^{2}=\tilde{\beta}+\left(\sqrt{\alpha^{(0)}}+\sqrt{\alpha^{(1)}}\right)^{2}$. Hence, with $\tilde{d}=\left[\left(d_{2}^{2}-\delta^{2}\right)\left(d_{1}^{2}-\delta^{2}\right)\right]^{1 / 2}$,

$$
\begin{aligned}
R_{2}^{(1-i)}(z) & =\frac{1-\alpha_{\min } / \alpha^{(1-i)}}{z}+\frac{\alpha_{\min }}{\alpha^{(1-i)}} \frac{2(z-\tilde{\beta}) / z}{z-\tilde{\beta}-\tilde{d}+\sqrt{z-d_{1}^{2}} \sqrt{z-d_{2}^{2}}} \\
& =\frac{\sqrt{b_{1} b_{2}}-\tilde{\beta}+\alpha^{(1-i)}-\alpha^{(i)}}{2 \alpha^{(1-i)} z}+\frac{1}{2 \pi \alpha^{(1-i)}} \int_{b_{1}}^{b_{2}} \frac{1}{z-x} \frac{\sqrt{b_{2}-x} \sqrt{x-b_{1}}}{x} d x
\end{aligned}
$$

if $i=r$, and

$$
\begin{aligned}
R_{2}^{(1-i)}(z) & =\frac{1-\alpha_{\min } / \alpha^{(1-i)}}{z-\tilde{\beta}}+\frac{\alpha_{\min }}{\alpha^{(1-i)}} \frac{2}{z-\tilde{\beta}-\tilde{d}+\sqrt{z-d_{1}^{2}} \sqrt{z-d_{2}^{2}}} \\
& =\frac{1-\alpha_{\min } / \alpha^{(1-i)}}{z-\tilde{\beta}}+\frac{1}{2 \pi \alpha^{(1-i)}} \int_{b_{1}}^{b_{2}} \frac{1}{z-x} \frac{\sqrt{b_{2}-x} \sqrt{x-b_{1}}}{x-\tilde{\beta}} d x
\end{aligned}
$$

if $i=1-r$, for $i=0,1$. Moreover,

$$
R_{3}(z)=\frac{1}{2 z}+\frac{1 / 2}{\sqrt{z-d_{1}^{2}} \sqrt{z-d_{2}^{2}}}=\frac{1}{2 z}+\frac{1}{2 \pi} \int_{b_{1}}^{b_{2}} \frac{1}{z-x} \frac{1}{\sqrt{x-b_{1}} \sqrt{b_{2}-x}} d x .
$$

The integral representations are derived from the results given in Lemma 2 and (4.1). Hence, the results of Theorem 5 can be given as 


$$
\begin{aligned}
\sum_{j=1}^{2 k+i} \tau_{2 k+i, j} f\left(\frac{z_{2 k+i, j}}{\lambda_{2 n}}\right) \rightarrow & \frac{\sqrt{b_{1} b_{2}}-\tilde{\beta}+\alpha^{(1-i)}-\alpha^{(i)}}{2 \alpha^{(1-i)}} f(0) \\
& +\frac{1}{2 \pi \alpha^{(1-i)}} \int_{b_{1}}^{b_{2}} f\left(x t^{\sigma}\right) \frac{\sqrt{b_{2}-x} \sqrt{x-b_{1}}}{x} d x, \quad \text { if } i=r, \\
\sum_{j=1}^{2 k+i} \tau_{2 k+i, j} f\left(\frac{z_{2 k+i, j}}{\lambda_{2 n}}\right) \rightarrow & \frac{\alpha^{(1-i)}-\alpha_{\min }}{\alpha^{(1-i)}} f\left(\tilde{\beta} t^{\sigma}\right) \\
& +\frac{1}{2 \pi \alpha^{(1-i)}} \int_{b_{1}}^{b_{2}} f\left(x t^{\sigma}\right) \frac{\sqrt{b_{2}-x} \sqrt{x-b_{1}}}{x-\tilde{\beta}} d x, \quad \text { if } i=1-r,
\end{aligned}
$$

for $i=0,1$, and

$$
\sum_{j=1}^{k} \tau_{k, j} f\left(\frac{z_{k, j}}{\lambda_{n}}\right) \rightarrow \frac{1}{2} f(0)+\frac{1}{2 \pi} \int_{0}^{1} \int_{b_{1}}^{b_{2}} f\left(x t^{\sigma}\right) \frac{1}{\sqrt{b_{2}-x} \sqrt{x-b_{1}}} d x d t .
$$

If $\alpha^{(0)}=\alpha^{(1)}$ and $\tilde{\beta}=0$ then $b_{1}$ is also zero.

Case 3. We assume that one of $\alpha^{(0)}$ or $\alpha^{(1)}$ is equal to zero, that is, for $r$ equal to 0 or 1 ,

$$
\alpha^{(r)}=0, \alpha^{(1-r)} \geq 0, \beta^{(r)}>0 \text { and } \beta^{(1-r)}>0 .
$$

In this case, again not covered by the previous theorems, $\alpha_{\text {min }}=0, d_{1}=d_{2}$ and $0<\beta \leq b_{1}=b_{2}$. Hence the results of Theorem 5 should be given as

$$
\sum_{j=1}^{2 k+i} \tau_{2 k+i, j} f\left(\frac{z_{2 k+i, j}}{\lambda_{2 n}}\right) \rightarrow \begin{cases}f\left(\beta^{(i)} t^{\sigma}\right), & \text { if } \quad i=r, \\ \frac{b_{2} \beta^{(1-i)}-\beta^{2}}{b_{2}^{2}-\beta^{2}} f\left(\left(\beta^{2} / b_{2}\right) t^{\sigma}\right)+\frac{b_{2}^{2}-b_{2} \beta^{(1-i)}}{b_{2}^{2}-\beta^{2}} f\left(b_{2} t^{\sigma}\right), & \text { if } i=1-r,\end{cases}
$$

for $i=0,1$, and

$$
\frac{1}{n} \sum_{j=1}^{n} f\left(\frac{z_{n, j}}{\lambda_{n}}\right) \rightarrow \frac{1}{2} \int_{0}^{1}\left\{f\left(\left(\beta^{2} / b_{2}\right) t^{\sigma}\right)+f\left(b_{2} t^{\sigma}\right)\right\} d t .
$$

6. Examples. We now consider some examples of polynomials that satisfy the recurrence relation (1.1) for which the coefficients satisfy the properties (3.1).

Example 1. Consider the monic polynomials $B_{n}^{H}$, defined by

$$
\int_{0}^{\infty} x^{-n+s} B_{n}^{H}(x) x^{-1 / 2} e^{-\frac{x+c^{2} / x}{2}} d x=0, \quad 0 \leq s \leq n-1, \quad n \geq 1,
$$

where $c>0$. These polynomials are related to the Hermite polynomials through a transformation given in [18]. The associated recurrence relation is given by

$$
B_{n+1}^{H}(z)=(z-c) B_{n}^{H}(z)-n z B_{n-1}^{H}(z), \quad n \geq 1,
$$

where $B_{0}^{H}(z)=1$ and $B_{1}^{H}(z)=z-c$. 
Condition (3.1) is valid with $\lambda_{n}=n(\sigma=1)$. Since $\beta^{(0)}=\beta^{(1)}=0$ and $\alpha^{(0)}=\alpha^{(1)}=1$, we are then in case 2 of the previous section with $b_{1}=0$.

In (3.3) we can also choose, for example, $A=4 \sqrt{1+c}$.

Thus, Theorem 1 should read. As $n \rightarrow \infty$ and $k / n \rightarrow t \in[0,1]$,

$$
\frac{1}{\lambda_{n}^{2}} \frac{B_{k}\left(\lambda_{n} z\right)}{B_{k-2}\left(\lambda_{n} z\right)} \rightarrow R_{1}(z, t)=t^{2} R_{1}(z / t)
$$

uniformly for $z$ on compact subsets of $\mathbb{C} \backslash[0,4 \sqrt{1+c}]$, where

$$
R_{1}(z)=\frac{1}{2}\left\{z^{2}-2 z+z \sqrt{z^{2}-4 z}\right\}
$$

From case 2 we have

$$
R_{2}^{(1-i)}(z)=\frac{2}{z+\sqrt{z(z-4)}}=\frac{1}{2 \pi} \int_{0}^{4} \frac{1}{z-x} \frac{\sqrt{4-x}}{\sqrt{x}} d x
$$

for $i=0,1$, and

$$
R_{3}(z)=\frac{1}{2 z}+\frac{1 / 2}{\sqrt{z} \sqrt{z-4}}=\frac{1}{2 z}+\frac{1}{2 \pi} \int_{0}^{4} \frac{1}{z-x} \frac{1}{\sqrt{x} \sqrt{4-x}} d x
$$

Hence, as $n \rightarrow \infty$ and $k / n \rightarrow t \in[0,1]$,

$$
\sum_{j=1}^{2 k+i} \tau_{2 k+i, j} f\left(\frac{z_{2 k+i, j}}{\lambda_{2 n}}\right) \rightarrow \frac{1}{2 \pi} \int_{0}^{4} f(x t) \frac{\sqrt{4-x}}{\sqrt{x}} d x
$$

for $i=0,1$, and

$$
\sum_{j=1}^{k} \tau_{k, j} f\left(\frac{z_{k, j}}{\lambda_{n}}\right) \rightarrow \frac{1}{2} f(0)+\frac{1}{2 \pi} \int_{0}^{1} \int_{0}^{4} f(x t) \frac{1}{\sqrt{4-x} \sqrt{x}} d x d t
$$

Example 2. We will now have a look at an example of polynomials with different limits for odd and even indices.

Van Assche [21] considered the two systems of orthogonal polynomials $\left\{C_{n}(x)\right\}_{n=0}^{\infty}$ and $\left\{D_{n}(x)\right\}_{n=0}^{\infty}$, with recurrence formulas given by

$$
\begin{aligned}
& C_{n+1}(w)=x C_{n}(w)-\tilde{\alpha}_{n+1}^{C} C_{n-1}(w), \\
& D_{n+1}(w)=x D_{n}(w)-\tilde{\alpha}_{n+1}^{D} D_{n-1}(w),
\end{aligned}
$$

with

$$
\begin{array}{ll}
\tilde{\alpha}_{2 m}^{C}=(2 m-1)^{2}, & \tilde{\alpha}_{2 m+1}^{C}=(2 m)^{2} \kappa^{2}, \\
\tilde{\alpha}_{2 m}^{D}=(2 m-1)^{2} \kappa^{2}, & \tilde{\alpha}_{2 m+1}^{D}=(2 m)^{2},
\end{array}
$$

where $\kappa$ is any positive real number. These are the famous Stieltjes-Carlitz polynomials connected with the Jacobi elliptic functions (see for example Chihara [3, Ch. V, Sec. 9]). 
As in [18], applying the transformation $w=\sqrt{z}-1 / \sqrt{z}$ on the systems $\left\{C_{n}(w)\right\}$ and $\left\{D_{n}(w)\right\}$ one can obtain two systems of polynomials $\left\{B_{n}^{C}(z)\right\}$ and $\left\{B_{n}^{D}(z)\right\}$, respectively, which satisfy three term recurrences relations of the type (1.1). Here, we will only consider the system $\left\{B_{n}^{D}(z)\right\}$, for which we have

$$
B_{n+1}^{D}(z)=(z-1) B_{n}^{D}(z)-\alpha_{n+1}^{D} z B_{n-1}^{D}(z),
$$

where $\alpha_{n}^{D}=\tilde{\alpha}_{n}^{D}$. Clearly these polynomials can be given by (1.2) with respect to some distribution function $\psi$ defined on $(0, \infty)$. From results provided in [21] one can show that if $0<\kappa<1$ then

$$
\psi(x)=\sum_{j=-\infty}^{\infty} \frac{q^{j}}{1+q^{2 j}} \frac{x_{j}}{1+x_{j}} U\left(x-x_{j}\right)
$$

where $x_{j}=\left[\sqrt{\left(j / K\left(\kappa^{2}\right)\right)^{2}+4}+j / K\left(\kappa^{2}\right)\right]^{2} / 4, x_{-j}=1 / x_{j}$ for $j \geq 0, q=$ $e^{-\pi K\left(1-\kappa^{2}\right) / K\left(\kappa^{2}\right)}$ and $K\left(\kappa^{2}\right)=\int_{0}^{\pi / 2}\left(1-\kappa^{2} \sin ^{2} \theta\right)^{-1 / 2} d \theta$. Again, $U$ is the Heaviside function.

For the coefficients of the recurrence relation (6.11) condition (3.1) holds with $\lambda_{n}=n^{2}(\sigma=2), \alpha^{(0)}=\kappa^{2}, \alpha^{(1)}=1$ and $\beta^{(0)}=\beta^{(1)}=0$. Hence, again we are in case 2 of the previous section, with $b_{1}=(1-\kappa)^{2}$ and $b_{2}=(1+\kappa)^{2}$

In (3.3) we can also choose $A=A(\kappa)$ to be equal to 4 if $0<\kappa<1$ or equal to $4 \kappa^{2}$ if $\kappa \geq 1$.

Thus, in Theorem 1 , we have as $n \rightarrow \infty$ and $k / n \rightarrow t \in[0,1]$,

$$
\frac{1}{n^{4}} \frac{B_{k}^{D}\left(n^{2} z\right)}{B_{k-2}^{D}\left(n^{2} z\right)} \rightarrow \frac{1}{2}\left\{z^{2}-\left(1+\kappa^{2}\right) t^{2} z+\sqrt{z^{2}-(\kappa+1)^{2} t^{2} z} \sqrt{z^{2}-(\kappa-1)^{2} t^{2} z}\right\}
$$

uniformly for $z$ on compact sets of $\mathbb{C} \backslash[0, A(\kappa)]$.

From case 2 we have, if $0<\kappa<1$,

$$
R_{2}^{(0)}(z)=\frac{1}{2 \pi \kappa^{2}} \int_{(1-\kappa)^{2}}^{(1+\kappa)^{2}} \frac{1}{z-x} \frac{\sqrt{(1+\kappa)^{2}-x} \sqrt{x-(1-\kappa)^{2}}}{x} d x
$$

and

$$
R_{2}^{(1)}(z)=\frac{1-\kappa^{2}}{z}+\frac{1}{2 \pi} \int_{(1-\kappa)^{2}}^{(1+\kappa)^{2}} \frac{1}{z-x} \frac{\sqrt{(1+\kappa)^{2}-x} \sqrt{x-(1-\kappa)^{2}}}{x} d x,
$$

and if $\kappa \geq 1$,

$$
R_{2}^{(0)}(z)=\frac{\kappa^{2}-1}{\kappa^{2} z}+\frac{1}{2 \pi \kappa^{2}} \int_{(1-\kappa)^{2}}^{(1+\kappa)^{2}} \frac{1}{z-x} \frac{\sqrt{(1+\kappa)^{2}-x} \sqrt{x-(1-\kappa)^{2}}}{x} d x
$$

and

$$
R_{2}^{(1)}(z)=\frac{1}{2 \pi} \int_{(1-\kappa)^{2}}^{(1+\kappa)^{2}} \frac{1}{z-x} \frac{\sqrt{(1+\kappa)^{2}-x} \sqrt{x-(1-\kappa)^{2}}}{x} d x .
$$

Moreover,

$$
R_{3}(z)=\frac{1 / 2}{z}+\frac{1}{2 \pi} \int_{0}^{1} \int_{(1-\kappa)^{2} t^{2}}^{(\kappa+1)^{2} t^{2}} \frac{1}{z-x} \frac{1}{\sqrt{x-(1-\kappa)^{2} t^{2}} \sqrt{(1+\kappa)^{2} t^{2}-x}} d x d t .
$$


Acknowledgement. The authors would like to thank both referees for the many valuable comments and references.

\section{REFERENCES}

[1] E.X.L. de Andrade, A. Sri Ranga and W. Van Assche, Asymptotics for polynomials satisfying a certain twin asymptotic periodic recurrence relation, Methods and Applications of Analysis, 6 (1999), pp. 535-548.

[2] G. Baxter, Polynomials defied by a difference system, J. Math. Anal. Appl., 2 (1961), pp. 223263.

[3] T. S. Chinara, An Introduction to Orthogonal Polynomials, Gordon and Breach, New York, 1978.

[4] P. Delsarte and Y. Genin, The split Levinson algorithm, IEEE Trans. Acoust. Speech Signal Process, 34 (1986), pp. 470-478.

[5] J. Dombrowski, Spectral properties of real parts of weighted shift operators, Indiana Univ. Math. J., 29 (1980), pp. 249-259.

[6] J. Dombrowski And G.H. Fricke, The absolute continuity of phase operators, Trans. Amer. Math. Soc., 213 (1975), pp. 363-372.

[7] J. Dombrowski And P. NeVAI, Orthogonal polynomials, measures and recurrence relations, SIAM J. Math. Anal., 17 (1986), pp. 752-759.

[8] E. Hendriksen and H. van Rossum, Orthogonal Laurent polynomials, Nederl. Akad. Wetensch. Indag. Math., 48 (1986), pp. 17-36.

[9] E. Hille, Analytic Function Theory, vol. 2, Blaisdell Publ. Co., Waltham, 1962.

[10] M.E.H. Ismail, Classical and Quantum Orthogonal Polynomials in one Variable, Cambridge Univ. Press, Cambridge, 2005.

[11] M.E.H. Ismail AND X. LI, Bounds on the extreme zeros of orthogonal polynomials, Proc. Amer. Math. Soc., 115 (1992), pp. 131-140.

[12] W.B. Jones, O. NuÅstad And W.J. Thron, Two-point Padé expansions for a family of analytic functions, J. Comput. Appl. Math., 9 (1983), pp. 105-123.

[13] W.B. Jones, W.J. Thron and H. WaAdeland, A strong Stieltjes moment problem, Trans. Amer. Math. Soc., 261 (1980), pp. 503-528.

[14] J.H. McCabe And J.A. Murphy, Continued fractions which correspond to power series expansions at two points, J. Inst. Math. Appl., 17 (1976), pp. 233-247.

[15] E.B. SAFF AND R.S. VARGA, Zero-free parabolic regions for sequences of polynomials, SIAM J. Math. Anal., 7 (1976), pp. 344-357.

[16] J. Shohat and J.D. Tamarkin, The Problem of Moments, revised edition, Amer. Math. Soc., Providence, 1950.

[17] A.P. Da Silva ANd A. SRi Ranga, Polynomials generated by a three term recurrence relation: bounds for complex zeros, Linear Algebra Appl., 397 (2005), pp. 299-324.

[18] A. SRI RANGA, Symmetric orthogonal polynomials and the associated orthogonal L-polynomials, Proc. Amer. Math. Soc., 123 (1995), pp. 3135-3141.

[19] A. Sri Ranga and L.C. Matioli, Bounds for the extreme zeros of polynomials generated by a certain recurrence relation, Rev. Mat. Estat., 14 (1996), pp. 113-120.

[20] W. Van Assche, Asymptotic properties of orthogonal polynomials from their recurrence formula I, J. Approx. Theory, 44 (1985), pp. 258-276.

[21] W. Van Assche, Asymptotic properties of orthogonal polynomials from their recurrence formula II, J. Approx. Theory, 52 (1988), pp. 322-338.

[22] H.S. WALL, Analytic Theory of Continued Fractions, Van Nostrand, Princeton, NJ, 1948.

[23] A. Zhedanov, On the polynomials orthogonal on regular polygons, J. Approx. Theory, 97 (1999), pp. 1-14. 
E.X.L. DE ANDRADE ET AL. 